\title{
Comparison of neck Range of Motion (ROM) and neck lordosis angle between spectacle users and non-spectacle users among first year undergraduates of the University of Peradeniya
}

Kumarasiri $\mathrm{KGSI}^{1}$ *, Jayasundara $\mathrm{BP}^{1}$, Kalahagahawaththa KWDP ${ }^{1}$, Lisana $\mathrm{MMF}^{1}$, Wijesinghe NP ${ }^{1}$, Karunathilaka KGLRAD ${ }^{1}$, Senarath MKID ${ }^{1}$, Sominanda A $^{1}$ ${ }^{I}$ Department of Physiotherapy, Faculty of Allied Health Sciences, University of Peradeniya ${ }^{2}$ Department of Anatomy, Faculty of Medicine, University of Peradeniya *sujanikumarasiri@gmail.com

Due to prism effect of glasses, objects are viewed in a different line of sight than the normal. Spectacle users might compensate this by lifting the chin or by leaning forward. Repetitive use of incorrect postures can become "daily habits" affecting the normal physiology and biomechanics of the neck. Present study was focused to determine the effect of spectacle wearing on neck ROM and lordosis angle and further to determine correlation between the cervical ROM and lordosis angle. A randomized sample of 100 first year undergraduates of University of Peradeniya (age $=19-24)$ were divided into a study group who wore spectacles $(n=50)$ and an age sex $\backslash$ matched control group who did not wear spectacles $(\mathrm{n}=50)$. The male:female ratio was 1:1. Cervical ROMs were measured using universal goniometer. Flexible ruler was used to measure the cervical lordosis. Significant differences were not found in cervical ROM ( $p>0.05$ ) and lordosis angle $(p>0.05)$ between spectacle users and non-users. Comparison of data across the subgroups; male wearers and non-wearers showed a significant difference $(p=0.012)$ among flexion angles. Weak positive correlations between forward flexion $(\mathrm{p}<0.001)$, right lateral flexion $(\mathrm{p}<0.05)$ and left lateral flexion $(p<0.05)$ among spectacle users and left neck rotation $(\mathrm{p}<0.05)$ among nonusers with the lordosis angle were found. The neck ROMs and lordosis angle between spectacle users and nonusers were found to be non-significant and therefore it can be concluded that neck ROM and lordosis are not affected by spectacle wearing in general. Positive correlations between neck ROM and lordosis angle among both groups were novel findings. 